\title{
Considerações sobre estudos de ambientes industriais e não industriais no Brasil: uma abordagem comparativa
}

\author{
Comments on studies of industrial \\ and non-industrial environments in Brazil: \\ a comparative approach
}

Adriana Gioda 1,2

Francisco Radler de Aquino Neto 1

\footnotetext{
1 Laboratório de Apoio ao Desenvolvimento Tecnológico, Instituto de Química, Centro de Tecnologia, Universidade Federal do Rio de Janeiro. C.P. 68563, Rio de Janeiro, RJ 21949-900, Brasil. ladetec@iq.ufrj.br

2 Departamento de Química, Universidade da Região de Joinville. Campus Universitário $s / n$, Joinville, SC 892001-972, Brasil. agioda@hotmail.com
}

\begin{abstract}
Air quality in industrial environments has been studied since the second half of the $20^{\text {th }}$ century. Well-documented results show that exposure to chemical pollutants through their consumption, production, and storage can damage workers' health. New research focusing on non-industrial environments has shown unexpected correlations between air quality and health effects. Artificial ventilation systems have been related to health-related complaints and a high rate of absenteeism. Symptoms related to air quality in non-industrial environments are recognized by the World Health Organization and encompassed under the so-called sick building syndrome (SBS). In Brazil there are few studies comparing indoor air quality and health. The present study is intended to map the studies already conducted in Brazil in order to encourage further research in this area, due to its importance for public health.
\end{abstract}

Key words Occupational Health; Indoor Air Pollution; Working Environment

Resumo A qualidade do ar em ambientes industriais tem sido palco de estudos desde a segunda metade do século XX. Resultados bem documentados apontaram que a exposição prolongada a poluentes químicos, proveniente do consumo, produção, armazenamento etc., pode causar danos à saúde do trabalhador. Recentemente, estudos direcionados a ambientes não industriais mostraram correlações surpreendentes entre a qualidade do ar e os efeitos causados à saúde. $O$ sistema de ventilação artificial tem sido a principal causa de reclamações e de um alto índice de absenteísmo. Os sintomas relacionados com a qualidade do ar em ambientes não industriais são reconhecidos pela Organização Mundial da Saúde que os identifica como "Síndrome dos Edifícios Doentes". No Brasil, há poucos estudos relacionando qualidade do ar em ambientes fechados e saúde. O presente trabalho pretende mapear as pesquisas já realizadas no País, visando, com isso, incentivar novos estudos nessa área, em virtude de sua importância para a saúde da população.

Palavras-chave Saúde Ocupacional; Poluição do Ar em Ambientes Fechados; Ambiente de Trabalho 


\section{Introdução}

A qualidade do ar em ambientes fechados tem sido motivo de pesquisa em todo o mundo devido aos danos causados quando em má qualidade. Inicialmente, as pesquisas direcionaram sua atenção para ambientes industriais, onde havia uma carga elevada de poluentes por causa da manipulação e exposição a substâncias tóxicas, na sua produção, consumo, armazenamento. Em virtude do aumento da industrialização em todo o mundo, principalmente a partir da segunda metade do século passado, muitos estudos foram realizados nessa área e foi possível relacionar os índices de poluição interna com efeitos adversos à saúde. A qualidade do ar nesses ambientes faz parte da saúde ocupacional.

No que concerne à qualidade do ar em ambientes não industriais, os estudos só tiveram início na década de 70, com o desenvolvimento de edifícios com maior eficiência no controle do consumo de energia. Estes dependiam do sistema de ventilação mecânica para circular de forma controlada o ar de renovação e para controlar temperatura e umidade, uma situação muitas vezes conflitante. O maior uso de materiais sintéticos na construção de edifícios, o aumento no número de trabalhadores e a automação do trabalho de escritórios, levando a maior estresse, contribuem para agravar os danos à saúde (Redlich et al., 1997). As doenças relacionadas com a qualidade do ar de interiores foram classificadas pela Organização Mundial da Saúde (OMS) como Síndrome dos Edifícios Doentes (SED), tal sua importância e prevalência, decorrentes da urbanização e verticalização dos ambientes internos.

\section{Saúde ocupacional}

A saúde ocupacional nasceu com a Revolução Industrial e é em grande parte fruto dos movimentos trabalhistas ingleses. Em 1957, a comissão mista da Organização Internacional do Trabalho (OIT) e OMS (Conferencia Internacional del Trabajo, 1959:25) definiu os objetivos sobre saúde ocupacional: "a saúde ocupacional tem como finalidade incentivar e manter o mais elevado nível de bem-estar físico, mental e social dos trabalhadores em todas as profissões; prevenir todo o prejuízo causado à saúde destes e pelas condições de seu trabalho; protegê-los em seu serviço contra os riscos resultantes da presença de agentes nocivos à sua saúde; colocar e manter o trabalhador em um emprego que convenha às suas aptidões fisiológicas e psi- cológicas e, em resumo, adaptar o trabalho ao homem e cada homem ao seu trabalho". Devese entender que a saúde, de acordo com a OMS, não é apenas a ausência de doença, mas, sim, o completo bem-estar físico, mental e social do indivíduo (Conferencia Internacional del Trabajo, 1959).

O assunto tomou a devida proporção apenas no final da década de 70, quando foi realizada a Assembléia Mundial de Saúde em 1979 (Resolução 32.14) (Pereira \& Otani, 1983) e 1980 (Resolução 33.31) (PAHO, 1983), que assinalava a necessidade urgente de se cuidar da melhor forma possível da saúde dos trabalhadores através de programas especiais. Também a Resolução XXXII, da XX Reunião do Conselho Diretor da Organização Pan-Americana da Saúde (OPAS) (PAHO, 1983) dava apoio aos programas de saúde ocupacional de acordo com a evolução industrial de cada país. A partir dessas reuniões, muitas outras se seguiram, buscando a melhoria das condições de trabalho e saúde ocupacional.

No Brasil, no final da década de 70, o sindicalismo operário paulista deu início a um processo de organização para a defesa da saúde dos trabalhadores. À época, o documento Saúde nas Fábricas colocava uma orientação para a organização sindical de trabalhadores e para o setor de saúde, a fim de construir alianças e estratégias em defesa da saúde. Em 1980, foi criado o Departamento Intersindical de Estudos em Saúde e Ambiente de Trabalho (DIESAT). Em São Paulo, em 1983, iniciam-se experiências relativas à saúde dos trabalhadores (Augusto \& Novaes, 1999).

Além dos acidentes de trabalho em ambientes industriais, a exposição prolongada a poluentes químicos procedentes das mais diversas fontes é um dos principais fatores no agravamento da saúde do trabalhador.

A Fundação Oswaldo Cruz (FIOCRUZ), Rio de Janeiro, criou o Centro de Estudos da Saúde do Trabalhador e Ecologia Humana (CESTEH) para realização de trabalhos de pesquisa relativos à saúde ocupacional.

\section{Síndrome dos Edifícios Doentes}

Os ambientes de escritórios foram, durante muitos anos, considerados isentos dos problemas de saúde ocupacional. Porém, no final dos anos 70, os pedidos de avaliação das condições ambientais em escritórios aos órgãos americanos ligados à saúde ocupacional (National Institute for Occupational Safety and Health NIOSH) aumentaram consideravelmente. O 
mesmo ocorreu na Europa, com similaridades entre as queixas (Ricard, 1998).

Os estudos da OMS (WHO, 1982) no início da década de 80 descreveram a relação entre os sintomas característicos da SED e a ventilação mecânica; porém, um estudo britânico foi mais longe e estabeleceu uma relação entre a existência dos sintomas e o sistema de ar condicionado (Ricard, 1998). Mas, foi a partir dos anos 90 que a SED tornou-se um conceito comum na literatura científica. O termo SED é usado para descrever situações nas quais os ocupantes de um determinado edifício experimentam efeitos adversos à saúde e ao conforto (Brickus \& Aquino Neto, 1999; WHO, 1984).

Atualmente sintomas relativos à SED não se restringem apenas a ambientes de escritórios. As pessoas permanecem $90 \%$ de seu tempo em locais fechados, isso inclui residências, escolas, hospitais, ou seja, todo tipo de ambiente não industrial. Nesses ambientes, ao contrário do que se imaginava, também há uma carga elevada de poluentes, típicas desses espaços, as quais podem afetar à saúde. O uso de combustíveis em residências, para cozimento e aquecimento, tem sido uma das maiores fontes de exposição a poluentes nesse tipo de ambiente. A combustão é geralmente ineficiente e, em condições de má ventilação, pode resultar em exposição elevada, que está associada a sintomas respiratórios e infecções, tanto em adultos (principalmente em mulheres), quanto em crianças (Husman, et al., 2002; Mehtal et al., 2002). A qualidade do ar em escolas também tem causado interesse no mundo científico devido ao longo tempo de permanência de crianças e jovens nesse tipo de ambiente. Má ventilação, com baixa taxa de troca de ar, além da falta de manutenção e limpeza insatisfatória das salas de aula, têm resultado em concentrações altas de dióxido de carbono e poeira causando sintomas da SED; com isso, há um aumento de alergias e asma, principalmente em crianças (Mi et al., 2002; Sahlberg et al., 2002). Hospitais e outros centros de saúde são tipos de meio ambiente complexos que requerem ventilação adequada para conforto e controle das emissões que possam ser prejudiciais a pacientes, funcionários e visitantes. A qualidade do ar nesse tipo de ambiente é mais crítica do que em outros locais fechados, devido ao aumento da susceptibilidade dos pacientes. Têm sido diagnosticados sintomas de SED em funcionários de hospitais e centros de saúde em virtude da exposição a vários agentes químicos e microbiológicos. Nesses ambientes são diagnosticadas principalmente infecções nosocomiais (Flatheim, 2002).
Por causa desses fatores, a poluição de interiores é hoje reconhecida como uma das maiores ameaças à saúde pública, de acordo com a OMS (WHO, 2000).

No Brasil, a saúde ocupacional tem se voltado para os problemas de maior vulto, que têm maior potencial de causar agravos à saúde do trabalhador e que ocorrem, em sua maioria, no ambiente industrial (Hoppe, 1999).

\section{Sintomas}

O diagnóstico da SED é eminentemente epidemiológico. De acordo com a OMS, na ausência de diagnóstico de patologias definidas, o diagnóstico se faz pela ocorrência de dois ou mais sintomas, que se sucedem pelo menos duas vezes na semana, no interior do prédio, e regridem quando a pessoa se afasta do ambiente em questão (Hoppe, 1999).

Os principais sintomas que devem ocorrer em um número estatisticamente significativo de usuários em um prédio são: irritação das mucosas, efeitos neurotóxicos, sintomas respiratórios e cutâneos e alteração dos sentidos. Sabe-se que uma série de fatores está associada à SED: contaminantes do ar (COVs, formaldeído, poeiras, fibras, asbestos); bioaerossóis (fungos, bactérias, vírus); fontes do meio ambiente; contaminantes gerados pela atividade humana; baixa taxa de renovação do ar, entre outros (Hoppe, 1999).

\section{Possíveis fontes}

No presente, nenhum fator ou grupo de fatores ambientais foi estabelecido como causa de SED, embora haja várias teorias. Por alguns anos, uma explicação popular era que compostos orgânicos voláteis atuando conjuntamente, embora presentes em baixos níveis, exerceriam um efeito tóxico. Essa idéia estava baseada nas propriedades irritantes e neurotóxicas desses compostos, que se manifestam quando os níveis de exposição são elevados. Ultimamente, maior atenção tem sido dada a contaminantes biológicos como fontes de poluentes, embora os dados obtidos até o momento sejam conflitantes. Alguns dados de efeitos sinérgicos em baixos níveis de exposição têm sido relatados (Hogue, 2000; Reisch, 1999; Roughi, 1999). Ventilação inadequada também é outro fator que está sendo investigado como possível causador de mal-estar entre ocupantes de ambientes fechados. Investigadores mais céticos acreditam que os fatores psicossociais e estresse também influenciem no bem-estar dos trabalhadores. Em vista da ausência de alguma teoria simples, 
a SED é considerada como de origem multifatorial, relacionada a vários fatores e graus de exposição (Redlich et al., 1997).

Recentemente, evidências experimentais têm mostrado que reações químicas entre ozônio e compostos orgânicos insaturados produzem espécies que causam fortes irritações nos olhos e vias respiratórias (Wolkoff \& Nielsen, 2001). Apesar disso, essas espécies não têm sido caracterizadas pelas amostragens e técnicas analíticas convencionais da Ciência do Meio Ambiente Interior. Estas estão voltadas para poucos compostos orgânicos voláteis e não voláteis, os quais poderiam igualmente ter efeitos biológicos potentes e estar relacionados a um aumento de queixas, além de representar um risco ao conforto e à saúde. Surge, portanto, a recomendação de uma análise mais abrangente de COVs, do que a de COVs "clássicos", como os definidos pela OMS (Wolkoff \& Nielsen, 2001). Não se pode afastar a hipótese, ainda de difícil comprovação, do sinergismo entre fatores de agressão, principalmente no caso de indivíduos sensíveis a substâncias químicas (Wolkoff \& Nielsen, 2001). O termo Sensibilidade Química Múltipla (Multiple Chemical Sensitivity - MCS) retrata como doença sem presumir um mecanismo ou etiologia particular. O número e tipo de pessoas que podem ser quimicamente sensíveis varia muito, e também são diferentes as conseqüências para cada indivíduo. A sensibilidade química provavelmente se desenvolve em dois estágios: (1) perda de tolerância específica seguida de exposição aguda ou crônica a vários agentes, tais como pesticidas, solventes ou ar contaminado em um edifício com SED, e (2) subseqüente "disparo" de sintomas por quantidades extremamente pequenas de substâncias químicas previamente toleráveis, drogas, alimentos e suas combinações (poluição por carros, fragrâncias, cafeína, álcool) (Miller, 1996).

De um modo geral, as fontes comuns de contaminantes no ar de interiores incluem, além da própria construção e decoração do ambiente interno, sua renovação e remodelamento. Tintas, carpetes, materiais de acabamento, adesivos, produtos de limpeza e máquinas de escritório liberam contaminantes para o ar interior sob a forma de gases, vapores ou partículas em suspensão. Curiosamente, apesar de quando novos emitirem uma maior e intensa carga de gases e COVs, esses materiais, com o uso e colonização por microorganismos, alteram seu perfil poluidor com o envelhecimento. A umidade relativa alta é uma das causas de crescimento de ácaros, fungos e bactérias. Também contribui a presença de nutrien- tes e locais para se desenvolverem (partículas, recessos onde estejam mais protegidos das práticas de limpeza e higienização). Materiais como carpetes podem servir como fontes secundárias, absorvendo COVs e particulados e liberando-os posteriormente; também são excelentes "criadouros" para microorganismos. Fatores físicos, tais como umidade relativa, barulho e luz, podem também contribuir para agravar os sintomas de SED. A segunda maior fonte dessa síndrome é o sistema de ventilação (Redlich et al., 1997).

\section{Custos}

Novas pesquisas têm mostrado que a qualidade do ar de interiores tem uma influência significativa e positiva na produtividade de trabalhadores de escritórios. A produtividade de funcionários, segundo Fanger (2000), é 6,5\% maior com boa qualidade do ar, além da redução de erros e de sintomas da SED. O estudo foi realizado na Dinamarca e na Suécia com resultados semelhantes (Fanger, 2000), e esse aumento deveria ser comparado com o custo de condicionamento do meio ambiente interior (MAI), o qual, para edifícios de escritórios em países desenvolvidos, é tipicamente $1 \%$ do custo do trabalho. Portanto, há um forte incentivo econômico para melhorar a qualidade do ar de interiores (Fanger, 2000).

Medidas de avaliação dos impactos à saúde causados pela poluição são muito complexas e os métodos disponíveis de análise econômica são muito rudimentares. Recentemente, entretanto, um progresso considerável tem sido feito especialmente em relação à poluição do ar. Embora a avaliação de morbidade seja muito importante para análise custo-benefício de programas de controle da qualidade do ar e para muitas outras áreas da atividade econômica, os estudos relevantes existentes são limitados no escopo e baseados inteiramente nos Estados Unidos (WB, 1998).

Fisk \& Rosenfeld (1997), com base em pesquisa bibliográfica, estimaram para os Estados Unidos um ganho anual de produtividade e economia de US\$ 6 a 14 bilhões pela redução de doenças respiratórias; US $\$ 2$ a 4 bilhões pela redução de alergia e asma e US\$ 10 a 30 bilhões pela redução da SED. Seppanen (1999) fez estimativas similares na Europa, calculando um custo anual, referente à qualidade do ar na Finlândia, em torno de 2,7 bilhões.

Estudos detalhados em anos recentes concluem que os casos de mortalidade e morbidade excessivos estão associados com altos níveis de exposição a material particulado (WB, 
1998), com óbvio custo social, mas também econômico. Por exemplo, no México, cada vida perdida devido à exposição a MPT foi avaliada em US\$ 75 mil, e no Brasil o custo de morte prematura foi estimado em US\$7.700 para São Paulo em 1989 e US\$ 25 mil para Cubatão em 1988. A grande diferença entre São Paulo e Cubatão deve-se à idade média com que a pessoa exposta morre (WB, 1998).

Ganhos na produtividade podem ser quantificados e poderiam servir como um forte estímulo para empregar medidas de conservação de energia que, simultaneamente, melhorem a qualidade do ambiente interior.

\section{Legislação}

Embora ainda não haja uma conscientização por parte da população da importância da qualidade do ar de interiores - QAI (ambientes não industriais), o governo está ficando alerta para o fato. Em meados de 1996, o Governo Federal Brasileiro proibiu o fumo em locais fechados de uso coletivo, demonstrando os primeiros sinais de importância da QAI. Também preocupado com o bem-estar dos passageiros, o Departamento de Aviação Civil (DAC) proibiu o fumo em todas as aeronaves brasileiras, independente do tempo de vôo. A fim de minimizar os efeitos à saúde, o Ministério da Saúde publicou a Portaria 3.523 de 28 de agosto de 1998 (Brasil, 1998) contendo Regulamento Técnico, cujo objetivo é "promover o estabelecimento de medidas referentes à limpeza dos sistemas de climatização e medidas específicas de padrões da qualidade do ar identificando poluentes de natureza física, química e biológica com suas respectivas fontes, visando a prevenção de riscos à saúde dos ocupantes desses ambientes". A Agência Nacional de Vigilância Sanitária (ANVISA), em decorrência da Portaria 3.523 (Brasil, 1998), publicou a Resolução 176 de 24 de outubro de 2000 (Brasil, 2000; atualmente em revisão) com algumas orientações técnicas sobre "Padrões Referenciais da Qualidade do Ar de Interiores em Ambientes Climatizados Artificialmente de Uso Público e Coletivo". Essa Resolução é revolucionária, pois define os parâmetros mínimos para uma boa qualidade do ar de interiores, como a concentração de $\mathrm{CO}_{2}$ e material particulado, temperatura, umidade relativa e velocidade do ar. Parâmetros mais complexos como COVs e aldeídos necessitam ser mais bem estudados para que sua influência sobre os ocupantes possa ser quantificada e padrões estabelecidos.

Aquino Neto \& Brickus (1999) sugeriram valores máximos para contaminantes físico-quí- micos presentes em ar de ambientes internos. Esses valores foram baseados em recomendações de organismos internacionais e nacionais adaptados à realidade brasileira. Nesse aspecto, características climáticas, sócio-econômicas, estruturais e geográficas do Brasil foram consideradas, bem como a matriz energética do País, nossa arquitetura, decoração e costumes. Nessa mesma linha de raciocínio, Kulcsar Neto \& Siqueira (2001) sugerem padrões referenciais para avaliar a qualidade do ar em interiores com base em parâmetros microbiológicos.

Regulamentações de saúde ocupacional relativa às atividades industriais continuam a ser uma atividade do Ministério do Trabalho, através do Decreto-Lei 5.452 de 1943, estabelecidas na NR-15 (Brasil, 1977). Esse Ministério tem baseado suas regulamentações nos valores de limite de tolerância publicados pela American Conference of Governamental Industrial Hygienists - ACGIH (Estados Unidos) (Frumkin \& Câmara, 1991). Os limites de exposição em mg/ $\mathrm{m}^{3}$ são baseados em $48 \mathrm{~h} /$ semana no Brasil e 40h/semana nos Estados Unidos. O modo como a NR-15 foi estabelecida gera alguns questionamentos sobre sua abrangência. Primeiro, com relação à equivalência de muitos padrões. O Brasil permite altas exposições a certas substâncias através de padrões permissíveis ou completa ausência de padrões. Esses padrões, embora sigam a ACGIH, não são atualizados como esta; com exceção do benzeno, os demais permanecem com os mesmos valores desde a criação da norma. Além disso, vários compostos carcinogênicos não são regulamentados no Brasil (Frumkin \& Câmara, 1991). Como ainda nem todos os compostos foram submetidos a todos os testes de toxidez necessários, é possível que, tanto no setor industrial, quanto em ambientes interiores, estejamos expostos a uma carga mais elevada de poluentes do que seria recomendável (Aquino Neto \& Brickus, 1999).

Os parâmetros de saúde ocupacional em ambientes industriais não definidos pela NR15 seguem outros padrões nacionais ambientais, como o Conselho Nacional do Meio Ambiente - CONAMA (Brasil, 1990). Apenas com fins comparativos, são utilizados parâmetros internacionais de saúde ocupacional, como os do NIOSH (1998), OSHA (1990) e OMS (WHO, 2000), e ambientais, como os da U.S. Environmental Protection Agency (USEPA, 1990). O mesmo é válido para avaliação do ar em ambientes não industriais. 


\section{Epidemiologia no Brasil}

Mesmo sabendo há muito que a exposição prolongada a agentes químicos causa problemas à saúde, poucos trabalhos foram encontrados na literatura no Brasil (revistas indexadas). Alguns trabalhos em locais fechados industriais são apresentados em níveis epidemiológicos, porém sem fazer uma correlação com a avaliação química do ar ambiente. Também são poucos os trabalhos relacionados com a QAI em ambientes não industriais, o que já era esperado, uma vez que essa preocupação é recente.

No contexto industrial, um dos primeiros trabalhos foi publicado por Belliboni et al. (1955), os quais estudaram dermatoses relacionadas a trabalhadores expostos a vários tipos de poluentes em diversas indústrias. $\mathrm{O}$ interesse dos autores foi despertado pela concentração já elevada e sempre crescente de indústrias em São Paulo e municípios vizinhos. Nessa oportunidade, foram examinados 2.138 operários de várias fábricas (cerâmica, cigarro, gêneros alimentícios, papel, cimento, iluminação, móveis de aço etc.) em plena atividade. Encontraram-se 221 casos de afecções cutâneas, das quais 73 de dermatoses profissionais e paraprofissionais, correspondendo a $3,5 \%$ dos indivíduos examinados e $33 \%$ das afecções cutâneas.

Mendes (1979) realizou um estudo no qual avaliou 3.440 pacientes masculinos internados por tuberculose pulmonar, obtendo 327 (9,5\%) de prováveis casos de silicose pulmonar (inalação de partículas contendo $\mathrm{SiO}_{2}$ ). Com base nesses dados, ele estimou, para o ano de 1977, 30 mil casos de portadores de silicose no Brasil. Esses pacientes eram trabalhadores de indústrias, principalmente de mineração, fundição, cerâmicas, vidro e de pedreiras. Nogueira et al. (1981) também realizaram trabalho semelhante em Jundiaí, São Paulo, e constataram casos de silicose em trabalhadores expostos, $76,6 \%$ dos quais apresentaram alterações radiológicas bem definidas.

Um outro estudo realizado por Mendes (1988) permitiu estimar que somente na Região Sudeste do Brasil existiam de 20 a $30 \mathrm{mil}$ portadores de silicose pulmonar nos seus diferentes estágios naquela década.

Outro poluente importante é o asbesto, o qual se constitui em um dos riscos ocupacionais/ambientais que maior preocupação vem trazendo aos países industrializados. Esse material tem sido utilizado na fabricação de mais de 3 mil produtos, principalmente na produção de telhas e caixas d'água, na indústria de fibrocimento e na indústria têxtil, na confec- ção de tecidos e papelões. A contaminação, através da inalação das fibras nos ambientes de trabalho, tem causado doenças respiratórias, tanto de vias aéreas, quanto parênquima pulmonar (Castro et al., 2001). No Município de Leme, São Paulo, foi detectada quantidade significativa de casos de asbestose (Mendes, 1988). Castro et al. (2001) fizeram uma avaliação respiratória em trabalhadores de indústria têxtil de amianto no Rio de Janeiro e as avaliações clínica, radiológica e funcional desses trabalhadores mostraram que $49 \%$ apresentavam tosse; $40 \%$, expectoração; $40 \%$, dispnéia e $28 \%$, chiado. Quanto à radiografia, $26 \%$ apresentaram alterações radiográficas compatíveis com a asbestose.

Morata et al. (1997) exploraram os efeitos de exposição ocupacional a solventes e barulho na audição de trabalhadores em gráficas de retrogravura na cidade de São Paulo. Os resultados encontrados sugerem que a exposição a tolueno tem um efeito tóxico no sistema auditivo.

Um dos poluentes mais estudados no Brasil em termos de saúde ocupacional é o benzeno. A cidade de Cubatão desencadeou esse estudo por causa de seu grande potencial poluidor gerado pelas indústrias da região. De acordo com Augusto \& Novaes (1999), no período de 1983 a 1995, mais de mil trabalhadores da siderúrgica do Município de Cubatão foram afastados do trabalho por apresentarem alterações hematológicas decorrentes da exposição ambiental e ocupacional ao benzeno. A Secretaria Municipal de Saúde de Volta Redonda (SMS-VR, 1999) registrou 688 casos de benzenismo nesse município no período entre 1984 a 1999. Entre as atividades econômicas relacionadas ao benzenismo destacam-se: metalurgia básica $(17,2 \%)$; fabricação de produtos de metal, exclusive máquinas e equipamentos $(37,8 \%)$; construção civil (32,3\%); serviços prestados principalmente às empresas $(7,2 \%)$ e outras $(5,5 \%)$. Fernícula et al. (1976) avaliaram a concentração de fenol em urina de trabalhadores expostos a benzeno em fábricas de calçado no Município de Franca, São Paulo. Os resultados mostraram uma concentração de fenol 5\% acima quando comparados com os de trabalhadores não expostos, o que indicava uma população susceptível a intoxicação crônica.

Faria et al. (1999) estudaram a relação entre câncer e industrialização através da evolução da mortalidade por essa doença na região da Baixada Santista, São Paulo, importante complexo industrial-portuário. Nesse trabalho, foram analisados 8.546 óbitos por câncer no período de 1980-1993. Os resultados apontaram 
para um excesso de mortalidade por câncer nessa região, o que estaria relacionado com a exposição a substâncias carcinogênicas (benzeno, cromo, derivados de alcatrão e óleos minerais, níquel, cádmio, tetracloreto de carbono, formaldeído, pentaclorofenol/dioxina e outros) presentes no processo de industrialização ocorrido nas últimas três décadas na localidade. Faria et al. (2000) também avaliaram a mortalidade por câncer no sistema nervoso nessa mesma região e período. Nesse caso, novamente foi comprovado um alto índice de mortalidade decorrente do meio ambiente e exposição ocupacional a substâncias orgânicas e inorgânicas.

Outro estudo avaliando o índice de casos de câncer em virtude da exposição ocupacional foi realizado na região metropolitana de São Paulo, a área mais industrializada do Brasil. A análise refere-se à exposição a asbestos, hidrocarbonetos policíclicos aromáticos, arsênico, poeira, níquel e cromo. Os resultados desse estudo revelaram riscos de câncer de pulmão para homens que trabalham em indústrias de maquinária e para trabalhadores de indústrias têxteis e olarias com longo tempo de exposição (Wunsch et al., 1995, 1998).

Silva et al. (2000) analisaram todos os óbitos (3.882) de militares ativos e inativos da marinha brasileira entre 1991 e 1995. Os resultados mostraram que certos tipos de câncer são mais comuns em militares do que na população em geral. Isso se deve às atividades ocupacionais dessa população que é exposta a carcinogênicos confirmados (asbesto, solventes orgânicos, óleos, radiações não ionizantes) e carcinogênicos potenciais (gases, diesel, fumaça, poeira de metais etc.). Além disso, é possível que a exposição seja de maior intensidade no interior de navios, onde as dimensões dos ambientes de trabalho são reduzidas e a ventilação nem sempre é adequada. Nesse caso, os resultados também indicaram que a mortalidade por tipo de câncer entre militares da marinha difere daquela da população geral de referência e se associa a certas funções.

Um dos primeiros trabalhos brasileiros relatados sobre SED foi estudado por Santos et al. (1992). Nesse trabalho, foram identificadas associações entre conforto ambiental e sinais e sintomas entre trabalhadores expostos a ambientes fechados, ou seja, ambientes com ventilação e climatização artificiais. Foram aplicados questionários a 312 trabalhadores bancários em dois edifícios na cidade de São Paulo. Na empresa com 95 trabalhadores, 18\% apresentaram sintomas gerais permanentes, $33 \%$ apresentaram sintomas de irritação de mem- brana mucosa permanentemente e $39 \%$ faltaram ao menos um dia de trabalho no espaço de seis meses por motivo de saúde. Na empresa com 217 trabalhadores, 9\% apresentaram sintomas gerais permanentes, $23 \%$ apresentaram sintomas de irritação de membrana mucosa permanentemente e $52 \%$ faltaram ao menos um dia de trabalho no espaço de seis meses por motivo de saúde. As percentagens elevadas indicam a relevância do problema em estudo.

Costa \& Brickus (2000) fizeram um estudo comparativo através de questionários sobre SED de funcionários em um shopping que utiliza ar condicionado central e em lojas de uma rua que utilizam ventilação natural em Niterói, Rio de Janeiro. Os resultados mostraram que houve uma grande incidência de sintomas associados à SED (maior número de queixas e sintomas de irritação) entre os funcionários que trabalham no shopping (ventilado artificialmente) quando comparados ao grupo de funcionários que trabalha em locais ventilados naturalmente. Esses dados também apontam para o fato de que os sintomas não são causados por um único fator, mas por uma combinação de fatores.

Machado et al. (2001) estudaram condições de saúde e trabalho em 23 bibliotecas de médio e grande porte no Rio de Janeiro e em outros estados do Brasil. Entre as informações obtidas, houve relato de $43 \%$ de casos de doenças relacionadas ao trabalho no último ano.

Em dezembro de 1996, foi avaliado o ar interior em uma biblioteca pública no Rio de Janeiro (Strauz et. al., 2001). A alta prevalência de queixas dermatológicas (50\%) e respiratórias $(38 \%)$ demonstraram que esse prédio estava com SED.

O total de mortes diretamente causadas pelo trabalho não é fácil de ser estimado. Em países com infra-estrutura de estatísticas vitais mais bem desenvolvida que o Brasil, essa dificuldade também existe. Há um número menor de relatos referentes à saúde ocupacional no Brasil quando comparados aos acidentes de trabalho. Então, é difícil avaliar o número de trabalhadores que sofrem algum dano à saúde devido à exposição a agentes químicos.

\section{Conclusões}

O levantamento de dados acima exposto mostrou que, embora há muito já sejam conhecidos os problemas de saúde ocupacional em face da exposição a poluentes químicos, poucos estudos foram realizados no Brasil abordando esses dois temas. A mesma tendência é obser- 
vada para SED, cujas pesquisas têm se limitado basicamente ao eixo Rio-São Paulo. Porém, se não forem tomadas as devidas providências, os casos de SED poderão aumentar, como mostram as experiências obtidas em outros países. Em virtude da já comprovada importância da qualidade do ar em ambientes fechados, espera-se que o País fique alerta a esse fato e, com isso, crie medidas que possam proteger a saúde e bem-estar da população.

\section{Referências}

AQUINO NETO, F. R. \& BRICKUS, L. S. R., 1999. Padrões referenciais para análise de resultados de qualidade físico-química do ar de interiores visando à saúde pública. Revista Brasindoor, 3:4-15.

AUGUSTO, L. G. S. \& NOVAES, T. C. P., 1999. Ação médico-social no caso do benzenismo em Cubatão, São Paulo: Uma abordagem interdisciplinar. Cadernos de Saúde Pública, 15:729-738.

BELliBONI, N.; ROTBERG, A.; PIMENTA, W. P. \& BEDRIKOW, B., 1955. Estudo preliminar das dermatoses industriais em SP. Arquivos da Faculdade de Higiene, 9:181-188.

BRASIL, 1990. Resolução no 03 de 28 de junho de 1990. Dispõe sobre padrões de qualidade do ar, previstos no PRONAR. Brasília: Diário Oficial da União, 22 ago.

BRASIL, 1998. Portaria 3523, de 28 de agosto de 1998. Procedimentos de verificação visual do estado de limpeza, remoção de sujidades por métodos físicos e manutenção do estado de integridade e eficiência de todos os componentes dos sistemas de climatização, para garantir a qualidade do ar de interiores e prevenção de riscos à saúde dos ocupantes de ambientes climatizados. Brasília: Diário Oficial da União, 31 ago.

BRASIL, 2000. Resolução 176, de 24 de outubro de 2000. Orientação Técnica sobre Padrões Referenciais de Qualidade do Ar Interior em ambientes climatizados artificialmente de uso público e coletivo. Brasília: Diário Oficial da União, 25 out.

BRICKUS, L. S. R. \& AQUINO NETO, F. R., 1999. A qualidade do ar de interiores e a química. Química Nova, 22:65-74.

CASTRO, H. A.; CANTO, P.; VICENTIN, G. \& MARINHO, A. C., 2001. Avaliação respiratória em trabalhadores de uma indústria têxtil de amianto, I Congresso Internacional de Qualidade do Ar Brasindoor. Revista Brasindoor, 5:34.

CONFERENCIA INTERNACIONAL DEL TRABAJO, 1959. La Organización de los Servicios de Medicina del Trabajo en los Lugares de Empleo. Geneva: Oficina Internacional del Trabajo.

COSTA, M. F. B. \& BRICKUS, L. S. R., 2000. Effect of ventilation systems on prevalence of symptoms associated with "Sick Buildings" in Brazilian commercial establishments. Archives of Environmental Health, 55:279-283.
FANGER, P. O., 2000. Indoor air quality in the 21 st century: Search for excellence. Indoor Air, 10:68-73.

FARIA, M. A. M.; ALMEIDA, J. W. R. \& ZANETTA, D. M. T., 1999. Mortalidade por câncer na região urbano-industrial da Baixada Santista, SP. Revista de Saúde Pública, 33:255-261.

FARIA, M. A. M.; ALMEIDA, J. W. R.; ZANETTA D. M. T. \& GATTAS, G. J. F., 2000. Nervous system cancer mortality in an industrialized area of Brazil 19801993. Arquivos Neuro-Psiquiátricos, 58:412-417.

FERNÍCULA, N. G. G.; WAKAMATSU, C. T.; MENDES, R. \& MORAES, E. C. F., 1976. Excreção urinária de fenol em trabalhadores de indústrias de calçados expostos ao benzeno. Revista de Saúde Pública, 10:327-333.

FISK, W. \& ROSENFELD, A., 1997. Estimatives of improved productivity and health from better indoor environments. International Journal of Indoor Air Quality and Climate, 7:158-172.

FLATHEIM, G., 2002. Hospital airborne infection prevention (HAIP). In: 9th International Conference on Indoor Air Quality and Climate, Proceedings, pp. 33-38, v. 2, Santa Cruz: Empire Grade.

FRUMKIN, H. \& CÂMARA, V. M., 1991. Occupational health and safety in Brazil. American Journal of Public Health, 81:1619-1624.

HOGUE, C., 2000. To MBTE, or not to MBTE. Chemical and Engineering News, 78:42-46.

HOPPE, L. F., 1999. Qualidade dos ambientes interiores e o papel da saúde ocupacional. In: I Encontro Paulista de Meio Ambiente e Controle da Qualidade do Ar de Interiores, Anais, pp. 43-51, São Paulo: Sociedade Brasileira de Meio Ambiente e Controle de Qualidade do Ar de Interiores.

HUSMAN, T.; MEKLIN, T.; VEPSALAINEN, A.; VAHTERISTO, M.; KOIVISTO, J.; HALLA-AHO, J.; HYVARINEN, A.; KOPONEM, V. \& NEVALAINEN, A., 2002, Respiratory infections among children in moisture damaged schools. In: 9th International Conference on Indoor Air Quality and Climate, Proceedings, pp. 485-490, Santa Cruz: Empire Grade.

KULCSAR NETO, F. \& SIQUEIRA, L. F. G., 2001. Padrões referenciais para análise de resultados de qualidade microbiológica do ar em interiores visando a saúde pública. Revista Brasileira de Saúde Ocupacional, 97/98:29-41.

MACHADO, J. M. H.; STRAUSZ, M. C.; BRICKUS, L. S. 
R. \& COSTA, M. F., 2001. Avaliação das condições de saúde e trabalho em ambientes com acervos documentais, I Congresso Internacional de Qualidade do Ar - Brasindoor, 25 a 28/03/01, Rio de Janeiro, RJ. Revista Brasindoor, 5:22.

MENDES, R., 1979. Estudo epidemiológico sobre a silicose pulmonar na Região Sudeste do Brasil, através de inquérito em pacientes internados em hospitais de Tisiologia. Revista de Saúde Pública, 13:7-19.

MENDES, R., 1988. O impacto dos efeitos da ocupação sobre a saúde de trabalhadores. I. Morbidade. Revista de Saúde Pública, 22:311-326.

MEHTAL, S.; SMITH, K. R.; BALAKRISHNAN, K.; SANKAR, S.; PADMAVATHI, R.; KUMAR, S. \& AKBAR, S., 2002. Using household characteristics to predict respirable particulate levels in rural households of Andhra Pradesh, India. In: 9th International Conference on Indoor Air Quality and Climate. Proceedings, pp. 506-601, Santa Cruz: Empire Grade.

MI, Y. H.; ELFMAN, L.; ERIKSSON, S.; JOHANSSON, M.; SMEDJE, G.; TAO, J.; MI, Y. L. \& NORBACK, D., 2002. Indoor allergens in schools: A comparison between Sweden and China. In: 9th International Conference on Indoor Air Quality and Climate, Proceedings, pp. 449-455, Santa Cruz: Empire Grade.

MILLER, C. S., 1996. Chemical sensitivity: Symptom, syndrome or mechanism for disease? Toxicology, 111:69-86.

MORATA T. C.; FIORINI A. C.; FISCHER, F. M.; COLACIOPPO S.; WALLINGFORD, K. M.; KRIEG, E. F.; DUNN, D. E.; GOZZOLI L, PADRÃO, M. A. \& CESAR, C. L. G., 1997. Toluene-induced hearing loss among rotogravure printing workers. Scandinavian Journal of Work, Environment and Health, 23:289-298.

MT (Ministério do Trabalho), 1994. Consolidação das Leis do Trabalho. São Paulo: Editora Atlas.

NIOSH, (National Institute for Occupational Safety and Health), 1994. NIOSH Pocket Guide to Chemical Hazards. Washington, DC: US Government Printing Office.

NOGUEIRA, D. P.; SOUZA, C. D.; BRÓLIO, R.; GARRAFA, N. M. \& SHIBATA, H., 1981. Ocorrência de silicose entre trabalhadores da indústria cerâmica de Jundiaí, SP, Brasil. Revista de Saúde Pública, 15:263-271.

OSHA (Occupational Safety and Health Administration), 1990. Occupational Exposure to Hazardous Chemicals in Laboratories. Washington, DC: Laboratory Standard.

PAHO (Pan American Health Organization), 1983. Program of Action on Workers Health. Washington DC: PAHO.

PEREIRA, J. C. R. \& OTANI, K. A., 1983. A saúde ocupacional como atividade de saúde pública: Uma experiência. In: II Congresso de Saúde Pública e I Congresso Nacional da Associação Brasileira de Pós-Graduação em Saúde Coletiva, Anais, p. 25, São Paulo: ABRASCO.

REDLICH, A.; SPARER, J. \& CULLEN, M. R., 1997. Sick-building syndrome. Lancet, 349:1013-1016.

REISCH, M. S., 1999. Paints \& coatings. Chemical and Engineering News, 77:22-33.
RICARD, I., 1998. Modelo de ambientes de escritórios: uma análise conceptual da "Síndrome dos Edifícios Doentes”. Revista Brasindoor, 3:28-29.

ROUGHI, A. M., 1999. Perfumes: A whiff of chemistry, Chemical and Engineering News, 77:38-46.

SAHLBERG, B.; SMEDJE, G. \& NORBACK, D., 2002. Sick building syndrome (SBS) among school employees in the county of uppsala, Sweden. In: $9^{\text {th }}$ International Conference on Indoor Air Quality and Climate, Proceedings, pp. 812-817, Santa Cruz: Empire Grade.

SANTOS, U. P.; RUMEL, D., MARTARELLO, N. A.; FERREIRA, C. S. W. \& MATOS, M. P., 1992. Síndrome dos edifícios doentes em bancários. Revista de Saúde Pública, 26:400-404.

SEPPANEN, O., 1999. Estimated cost of indoor climate in Finnish buildings. In: The 8th International Conference On Indoor Air Quality and Climate, Anais, pp. 9-13, London: Construction Research Communication.

SILVA, M.; SANTANA, V. S. \& LOOMIS, D., 2000. Mortalidade por câncer em militares da Marinha do Brasil. Revista de Saúde Pública, 34:373-379.

SMS-VR (Secretaria Municipal de Saúde de Volta Redonda), 1999. Relatório sobre os Casos de Benzenismo no Município de Volta Redonda, Sistema Único de Saúde. Volta Redonda: Prefeitura Municipal de Volta Redonda.

STRAUSZ, M. C.; MACHADO, J. M. L. \& BRICKUS, L. S. R., 2001. Aplicação de metodologia de análise de acidentes em um caso de síndrome do edifício doente, I Congresso Internacional de Qualidade do $\mathrm{Ar}$ - Brasindoor, 25 a 28/03/01, Rio de Janeiro, RJ. Revista Brasindoor, 5:26.

USEPA (U.S. Environmental Protection Agency), 1990. Technical Assistance Document for Sampling and Analysis of Toxic Organic Compounds in Ambient Air. Washington DC: USEPA.

WHO (World Health Organization), 1982. Indoor Air Pollutants: Exposure and Health Effects. Report WHO Meeting, Nordlingen: WHO.

WHO (World Health Organization), 1984. Indoor Air Quality Research. Stockholm: WHO.

WHO (World Health Organization), 2000. The Right to Health Indoor Air. Bilthoven: WHO.

WOLKOFF, P. \& NIELSEN, G. D., 2001. Organic compounds in indoor air - Their relevance for perceived indoor air quality? Atmospheric Environment, 35:4407-4417.

WB (World Bank), 1998. Pollution Prevention Abatement Handbook 1998: Toward Cleaner Production. Washington, DC: WB.

WUNSCH, V.; MAGALDI, C.; NAKAO, N. \& MONCAU, J. E. C., 1995. Industrial work and lung-cancer. Revista de Saúde Pública, 29:166-176.

WUNSCH, V.; MONCAU, J. E.; MIRABELLI, D. \& BOFFETTA, P., 1998. Occupational risk factors of lung cancer in Sao Paulo, Brazil. Scandinavia Journal of Work Environment and Health, 24:118-124.

Recebido em 6 de maio de 2002

Versão final reapresentada em 19 de novembro de 2002 Aprovado em 21 de maio de 2003 